\title{
Listeria monocytogenes: a bacterial pathogen to hit on the SUMO pathway
}

\author{
Simona Citro ${ }^{1}$, Susanna Chiocca ${ }^{1}$ \\ ${ }^{1}$ European Institute of Oncology, Department of Experimental Oncology, IFOM-IEO Campus, Via Adamello 16, 20139 Milan, Italy \\ Cell Research (2010) 20:738-740. doi:10.1038/cr.2010.76; published online 8 June 2010
}

Attachment of ubiquitin (Ub) or ubiquitin-like (Ubl) modifiers, such as SUMO, is a reversible post-translational modification that regulates the fate and function of proteins.

During infectious disease in plants and animals, pathogens secrete proteins or small molecules inside their hosts to produce proper conditions for their growth. Bacterial and viral pathogens exploit cellular pathways for their own advantage; components of the ubiquitination machinery are recurrent targets of pathogen manipulation.

Frequently, viruses encode proteins that highjack cellular E3 ligases in order to eliminate unwanted cellular proteins, but they can also inhibit E3 ligases to prevent specific protein degradation and use the system to control the level of expression of their own proteins [1].

Similar to ubiquitin, the SUMO pathway is also a target for pathogens (review [2], Figure 1). We have shown that the early gene product of the avian CELO adenovirus, Gam1, induces an overall inhibition of protein sumoylation in the cell by degrading the SUMO E1 enzyme through recruitment of endogenous cellular components of ubiquitin E3 ligases [3, 4]. In a further extension of the importance of the SUMO pathway during the infectious

Correspondence: Susanna Chiocca

Tel: +39-02-57489835; Fax: +39-02-94375990

E-mail: susanna.chiocca@ifom-ieo-campus.it process by viruses, a recently published report [5] shows the original ability of a bacterial factor to induce alteration of the protein sumoylation status in the host cell.

Covalent conjugation of $\mathrm{Ub} / \mathrm{Ubl}$ to their substrates requires an analogous enzymatic cascade comprising the sequential action of three enzymes [6]: a modifier activating enzyme (E1), one of several modifier carrier enzymes (E2s), and a member of the large and diverse group ligases (E3s), which enhance conjugation to the target and determine target specificity. Whereas the ubiquitin cascade presents different enzymes with E2 activity, for the sumoylation machinery only one $\mathrm{E} 2$ enzyme has been identified so far, UBC9 and one E1 enzyme (the SAE1/SAE2 heterodimer). Gam1 is the only viral protein that has been shown to interfere with the sumoylation pathway of the host cell, by direct inactivation and proteasome dependent degradation of SAE1/SAE2. Moreover, by a still unknown mechanism, Gam 1 overexpression also leads to UBC9 degradation. Ribet et al. asked whether the bacterial infection mediated by Listeria monocytogenes, the pathogen responsible for human listeriosis, was also able to alter the host protein sumoylation for the onset and/or propagation of the infectious process.

Listeria monocytogenes is a Grampositive bacterium responsible for severe food-borne diseases such as meningitis, meningo-encephalitis, materno- fetal and perinatal infection.

These syndromes are due to the ability of the bacterium to cross the intestinal, blood-brain and materno-fetal host barriers, to the capacity to resist intracellular killing by macrophages and to invade and replicate into normally nonphagocitic cells [7]. During infection L. monocytogenes manipulates many cellular machineries in the host, including the host-epigenetic machinery, to its own benefit. Thus, since L. monocytogenes triggers posttranslational modifications such as phosphorylation of E-cadherin followed by its ubiquitination [8], Ribet et al. asked whether Listeria was also able to modify the host cell sumoylation status upon infection. They found that cells infected by $L$. monocytogenes, but not by the non-pathogenic L. innocua, displayed a reduction in SUMO-conjugated proteins triggered by the secreted pore-forming toxin Listeriolysin O (LLO). Surprisingly, they found that this effect on the sumoylation machinery was due to a dramatic reduction of the E2 enzyme UBC9 in the cells. Differently from Gam1, which induces degradation of both UBC9 and SAE1/SAE2, LLO induces only UBC9 degradation in a proteasome-independent manner but dependent on its ability to bind the cellular membranes and to form pores. Similar to LLO, other toxins with pore-forming properties, such as PFO and PLY encoded by Clostridium perfringes and Streptococcus pneumonie, respectively, 
were also able to induce UBC9 degradation, suggesting that other pathogenic bacteria are able to target the SUMO pathway in a mechanism similar to the one used by Listeria.

Deubiquitination is also an important viral target. Several viral proteins are known to interact with cellular deubiquitinating enzymes (DUBs) and some viral proteins possess deubiquitinating activity themselves. Thus, not only modification of ubiquitinated substrates during viral infection is a mainstream event, but examples of viruses that encode their own ubiquitin ligases as well as deubiquitinating enzymes are rapidly increasing [1]. Viruses are known to interfere with the SUMO pathway by inducing promyelocytic leukaemia (PML) desumoylation followed by disruption of the subnuclear structures known as the PML oncogenic domains (PODs), through mechanisms dependent [9] or independent on SUMO proteases [10] (Figure1). Ribet et al. showed that the LLO-mediated UBC9 degradation involves the activity of an aspartylprotease, concluding that LLO triggers global protein desumoylation in cells simultaneously with the degradation of some SUMO-conjugated proteins. This mechanism can be explained considering that SUMO-conjugation increases the stability of some substrates, and thus once desumoylated they are less stable. To further investigate this downstream effect, Ribet et al. looked at the effect of LLO on the TGF $\beta$ response of the cell, where sumoylation stabilizes SMAD4, a central intracellular transducer for TGF $\beta$ signaling [11]. They observed that both LLO incubation and Listeria infection promoted a decrease of SMAD4 in the cells, an effect counteracted by the overexpression of SUMO. Moreover, the TGF $\beta$ response in cells infected with Listeria was also impaired, suggesting that loss of SUMO conjugation affects TGF $\beta$ signaling in infected cells. This effect might be beneficial to Listeria replication since TGF $\beta$ has been proposed to be protective in host resistance against $L$. monocytogenes [12]. Thus, a way to counteract bacteria infection would be to increase the level of sumoylated protein in the cells. Ribet et al. approached this question by showing that overexpression of SUMO in infected cells decreases the number of intracellular bacteria, suggesting a

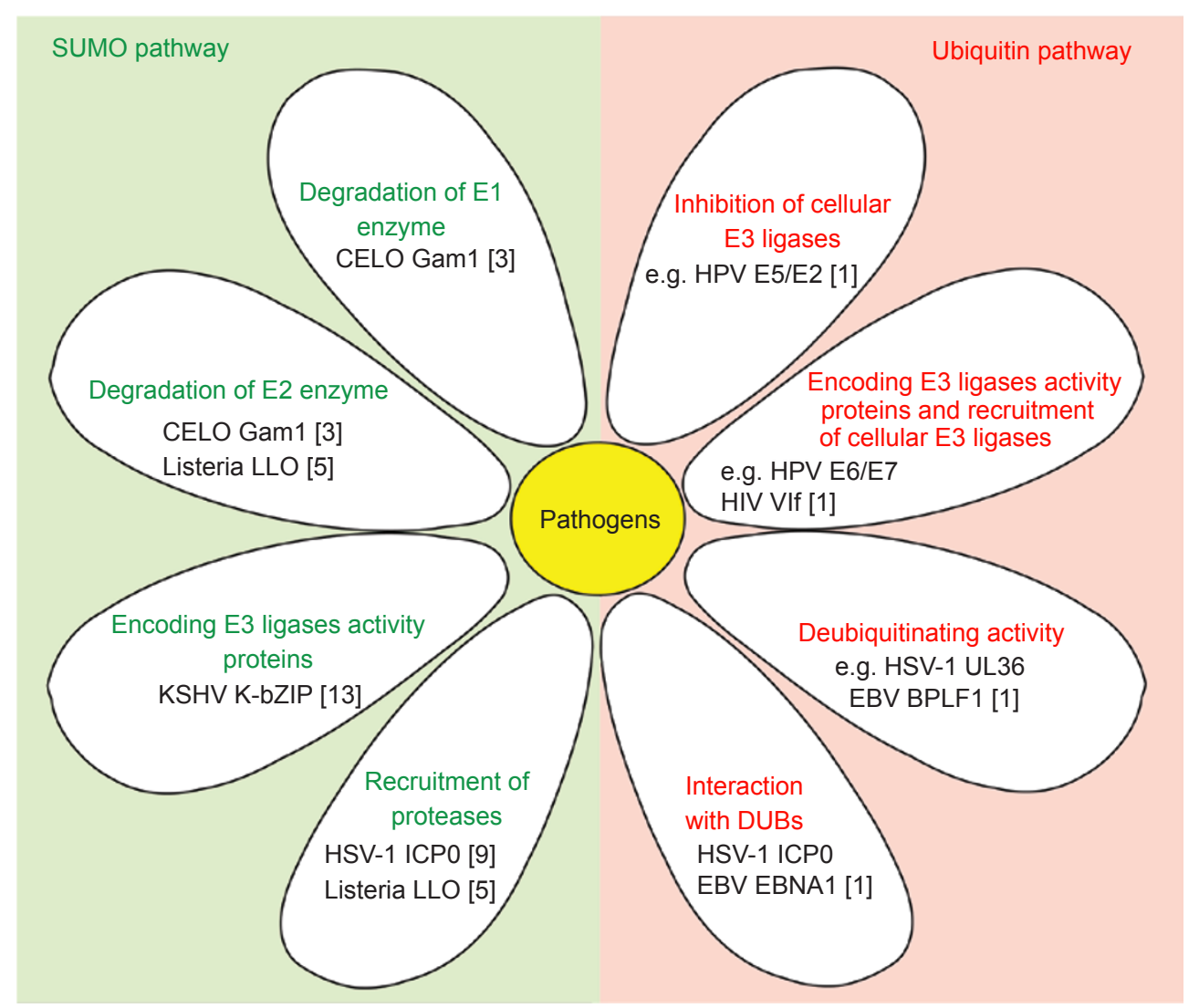

Figure 1 Pathogens exploitation of host SUMO and ubiquitin pathways: viruses and bacteria have developed similar strategies to avoid or enhance the ubiquitination and sumoylation machineries, mainly by encoding in their own genome proteins belonging to these networks or by hijacking cellular enzymes. Examples in the figure include viral proteins interacting with both the ubiquitin [1] and the SUMO pathways [3, 5, 9, 13]. 
protective role of sumoylation against bacterial invasion and/or replication. In conclusion, the study of Ribet et al. has uncovered a previously unknown mechanism of Listeria: Listeria promotes its infection by targeting the SUMO pathway. Unprecedented, they show that the SUMO E2 enzyme, UBC9, is a target for protein degradation mediated by a bacterial protein. It seems that the infectious machinery engaged by Listeria causes an impairment in the overall SUMO conjugation that can be not only due to the decrease of UBC9 expression but also to the activation of a deconjugation pathway, probably related to the recruitment of some proteases. Moreover, since Listeria-mediated degradation of UBC9 is a proteasome-independent event, it could be related to the activation of some proteases that might use it as a substrate. A broader investigation of this pathway will be essential to understand the mechanism that Listeria and other pathogens use to promote their infection. Nevertheless, this work underscores the importance of studying SUMO pathway components as potential drug targets for anti-pathogen therapies.

\section{References}

1 Isaacson MK, Ploegh HL. Ubiquitination, ubiquitin-like modifiers, and deubiquitination in viral infection. Cell Host Microbe 2009; 5:559-570.

2 Boggio R, Chiocca S. Viruses and sumoylation: recent highlights. Curr Opin Microbiol 2006; 9:430-436.

3 Boggio R, Colombo R, Hay RT, et al. A mechanism for inhibiting the SUMO pathway. Mol Cell 2004; 16:549-561.

4 Boggio R, Passafaro A, Chiocca S. Targeting SUMO E1 to ubiquitin ligases: a viral strategy to counteract sumoylation. J Biol Chem 2007; 282:1537615382.

5 Ribet D, Hamon M, Gouin E, et al. Listeria monocytogenes impairs SUMOylation for efficient infection. $\mathrm{Na}$ ture 2010; 464:1192-1195.

6 Passmore LA, Barford D. Getting into position: the catalytic mechanisms of protein ubiquitylation. Biochem $J$ 2004; 379 (Pt 3):513-525.

7 Cossart P, Toledo-Arana A. Listeria monocytogenes, a unique model in infection biology: an overview. Microbes
Infect 2008; 10:1041-1050.

8 Bonazzi M, Veiga E, Pizarro-Cerda J, et al. Successive post-translational modifications of E-cadherin are required for InlA-mediated internalization of Listeria monocytogenes. Cell Microbiol 2008; 10:2208-2222.

9 Bailey D, O'Hare P. Herpes simplex virus 1 ICP0 co-localizes with a SUMOspecific protease. J Gen Virol 2002; 83 (Pt 12):2951-2964.

10 Kang H, Kim ET, Lee HR, et al. Inhibition of SUMO-independent PML oligomerization by the human cytomegalovirus IE1 protein. J Gen Virol 2006; 87 (Pt 8):2181-2190.

11 Lin $\mathrm{X}$, Liang $\mathrm{M}$, Liang $\mathrm{YY}$, et al. SUMO-1/Ubc9 promotes nuclear accumulation and metabolic stability of tumor suppressor Smad4. J Biol Chem 2003; 278:31043-31048.

12 Nakane A, Asano M, Sasaki S, et al. Transforming growth factor beta is protective in host resistance against Listeria monocytogenes infection in mice. Infect Immun 1996; 64:3901-3904.

13 Chang PC, Izumiya Y, Wu CY, et al. Kaposi's sarcoma-associated herpesvirus (KSHV) encodes a SUMO E3 ligase that is SIM-dependent and SUMO-2/3-specific. J Biol Chem 2010; 285:5266-5273. 